\title{
List of vascular plants endemic to Britain, Ireland and the Channel Islands 2020
}

\author{
Timothy C.G. Rich \\ Cardiff, U.K.
}

\begin{abstract}
Corresponding author: Tim Rich: tim_rich@sky.com
\end{abstract}
This pdf constitutes the Version of Record published on $31^{\text {st }}$ August 2020

\begin{abstract}
A list of 804 plants endemic to Britain, Ireland and the Channel Islands is broken down by country. There are 659 taxa endemic to Britain, 20 to Ireland and three to the Channel Islands. There are 25 endemic sexual species and 26 sexual subspecies, the remainder are mostly critical apomictic taxa. Fifteen endemics (2\%) are certainly or probably extinct in the wild.
\end{abstract}

Keywords: England; Northern Ireland; Republic of Ireland; Scotland; Wales.

\section{Introduction}

This note provides a list of vascular plants endemic to Britain, Ireland and the Channel Islands, updating the lists in Rich et al. (1999), Dines (2008), Stroh et al. (2014) and Wyse Jackson et al. (2016). The list includes endemics of subspecific rank or above, but excludes infraspecific taxa of lower rank and hybrids (for the latter, see Stace et al., 2015). There are, of course, different taxonomic views on some of the taxa included.

Nomenclature, taxonomic rank and endemic status follows Stace (2019), except for Hieracium (Sell \& Murrell, 2006; McCosh \& Rich, 2018), Ranunculus auricomus group (A. C. Leslie in Sell \& Murrell, 2018), Rubus (Edees \& Newton, 1988; Newton \& Randall, 2004; Kurtto \& Weber, 2009; Kurtto et al. 2010, and recent papers), Taraxacum (Dudman \& Richards, 1997; Kirschner \& Štepànek, 1998 and recent papers) and UImus (Sell \& Murrell, 2018). Ulmus is included with some reservations, as many taxa are largely vegetative clones which may occasionally reproduce sexually and hence may not merit species status (cf. Stace, 2019). There are two recent endemics described from Scotland, A/chemilla sciura and the diploid Dryopteris affinis subsp. cluthensis (Lynes, 2019; Church et al., 2019). Two recentlydescribed Scottish Alchemilla endemics (Hogarth, 2020) require valid publication before being accepted.

I differ from Stace (2019) in recognising Cochlearia atlantica but not accepting C. micacea or C. officinalis subsp. scotica, or Anthyllis vulneraria subsp. corbierei which is simple genetic variation (Rich, 2006). Dryopteris kerryensis is here accepted at species rank and Sorbus avonensis in now also treated as a species as the population forms a distinct clone in the Avon Gorge of about 42 individuals ( $\mathrm{L}$. Houston, unpublished data, pers. comm. 2020). 
The political regions to which species are endemic are given to indicate geographic responsibilities and priorities; these are Channel Islands, England, Isle of Man, Scotland or Wales or for any combination of these as Britain, and Northern Ireland or the Republic of Ireland or where both as Ireland, and where in both Britain and Ireland as Britain \& Ireland. Fifteen endemics are certainly or probably extinct in the wild but there is little recent data for many other taxa which could also be extinct.

The list is given in Appendix 1 and the number of taxa endemic to each region is summarised in Tab. 1 . The endemics are mostly critical apomictic taxa; Alchemilla (2), Dryopteris (1), Hieracium (332), Limonium (12), Ranunculus auricomus group (58), Rubus (194), Sorbus (38) and Taraxacum (53). There are 25 sexual species and 26 sexual subspecies.

Table 1. Number of endemics in each region

\begin{tabular}{|c|c|c|}
\hline Geographic Region & No. endemics & Total \\
\hline Channel Islands & 3 & \\
\hline England & 287 & \\
\hline Isle of Man & 0 & \\
\hline Scotland & 189 & \\
\hline Wales & 59 & \\
\hline Britain & 121 & \\
\hline Total for Britain & & 659 \\
\hline Northern Ireland & 0 & \\
\hline Republic of Ireland & 14 & \\
\hline Ireland & 6 & \\
\hline Total for Ireland & & 20 \\
\hline Britain \& Ireland & 125 & \\
\hline Overall Total & & 804 \\
\hline
\end{tabular}

\section{Acknowledgements}

I would like to thank Ian Denholm, Hendrik Devriese, Dave Earl, Libby Houston, Alan Leslie, David McCosh, Rob Randall, John Richards, Fred Rumsey, Clive Stace, Abraham van der Beek and Mike Wyse Jackson.

\section{References}

Church, A.R., Evans, A.J., Golding, R., Rumsey, F.J. \& Viane, R.L.L. 2019. Dryopteris affinis subsp. cluthensis. A new taxon in the Dryopteris affinis complex (Dryopteridaceae). Fern Gazette, 21: 85-95.

Dines, T. 2008. A vascular plant Red Data List for Wales. Salisbury: Plantlife International.

Dudman, A. \& Richards, A.J. 1997. Dandelions of Great Britain and Ireland. London: Botanical Society of the British Isles.

Edees, E.S. \& Newton, A. 1988. Brambles of the British Isles. London: Ray Society. Hogarth, B.G. 2020. Alchemilla A field guide to the lady's-mantles of the British Isles. Scotland: Amenta Publishing.

Kirschner, J. \& Štepànek, J. 1998. A monograph of Taraxacum sect. Palustria. Pruhonice: Institute of Botany, Academy of Sciences of Czech Republic. 
Kurtto, A. \& Weber, H.E. 2009. Rubus. In: Kurtto, A., ed.: Rosaceae. Euro+Med Plantbase - the information resource for Euro-Mediterranean plant diversity. [accessed April 2020]. Available at http://ww2.bgbm.org/EuroPlusMed/.

Kurtto, A. \& Weber, H.E., Lampinen, R. \& Sennikov, A.N., eds. 2010. Atlas Flora Europaeae. Distribution of Vascular plants in Europe. 15. Rosaceae (Rubus). Helsinki: The Committee for Mapping the Flora of Europe \& Societas Biologica Fennica Vanamo.

Lynes, M. 2019. Alchemilla sciura (Rosaceae), a new species of Lady's-mantle. British \& Irish Botany 1: 335-341.

McCosh, D.J. \& Rich, T.C.G. 2018. Atlas of British and Irish Hawkweeds (Pilosella Hill and Hieracium L.). 2nd ed. Harpenden: Botanical Society of Britain and Ireland.

Newton, A. \& Randall, R.D. 2004. Atlas of British and Irish Brambles. London: Botanical Society of the British Isles.

Rich, T.C.G. 2006. A simple genetic base for the spreading and appressed hair types in Anthyllis vulneraria L. (Fabaceae). Watsonia, 26: 69.

Rich, T.C.G., Hutchinson, G., Randall, R.D. \& Ellis, R.G. 1999. List of plants endemic to the British Isles. BSBI News, 80: 23-27.

Sell, P.D. \& Murrell, G. 2006. Flora of Great Britain and Ireland. Volume 4. Cambridge: Cambridge University Press.

Sell, P.D. \& Murrell, G. 2018. Flora of Great Britain and Ireland. Volume 1. Cambridge: Cambridge University Press.

Stace, C.A., Preston, C.D. \& Pearman, D.A. 2015. Hybrid flora of the British Isles. Bristol: Botanical Society of Britain and Ireland.

Stace, C.A. 2019. New flora of the British Isles. 4th ed. Middlewood Green, Suffolk: C \& M Floristics.

Stroh, P.A., Leach, S. J., August, T.A., Walker, K.J., Pearman, D.A., Rumsey, F.J., Harrower, C.A., Fay, M.F., Martin, J.P., Pankhurst, T., Preston, C.D. \& Taylor, I. 2014. A Vascular Plant Red List for England. Bristol: Botanical Society of Britain and Ireland.

Wyse Jackson, M., FitzPatrick, Ú., Cole, E., Jebb, M., McFerran, D., Sheehy Skeffington, M. \& Wright, M. 2016. Ireland Red List No. 10: Vascular Plants. Dublin: National Parks and Wildlife Service, Department of Arts, Heritage, Regional, Rural and Gaeltacht Affairs.

Copyright retained by author(s). Published by BSBI under the terms of the Creative Commons Attribution 4.0 International Public License.

ISSN: 2632-4970

https://doi.org/10.33928/bib.2020.02.169 


\section{Appendix 1. List of vascular plants endemic to Britain, Ireland and the Channel Islands broken down by country.}

\begin{tabular}{|l|l|}
\hline Species & Distribution summary \\
\hline Alchemilla minima & England \\
\hline Alchemilla sciura & Scotland \\
\hline Arenaria ciliata subsp. hibernica & Ireland \\
\hline Arenaria norvegica subsp. anglica & England \\
\hline Bromus interruptus & England (Extinct) \\
\hline Calamagrostis scotica & Scotland \\
\hline Centaurium intermedium & England \\
\hline Centaurium tenuiflorum subsp. & England but further studies in NW \\
anglicum & Europe required \\
\hline Cerastium fontanum subsp. scoticum & Scotland \\
\hline Cochlearia atlantica & Britain \& Ireland \\
\hline Coincya monensis subsp. monensis & Britain \\
\hline Coincya wrightii & England \\
\hline Cotoneaster cambricus & Wales \\
\hline Dactylorhiza incarnata subsp. coccinea & Britain \& Ireland but further studies in \\
& NW Europe required \\
\hline Dactylorhiza incarnata subsp. gemmana & Britain \& Ireland but further studies in \\
& NW Europe required \\
\hline Dactylorhiza incarnata subsp. pulchella & Britain \& Ireland but further studies in \\
& NW Europe required \\
\hline Dactylorhiza kerryensis & Republic of Ireland \\
\hline Dactylorhiza traunsteinerioides & Britain \& Ireland \\
\hline Dryopteris affinis subsp. cluthensis & Scotland \\
\hline Dryopteris kerryensis & Britain \& Ireland \\
\hline Epipactis dunensis & Britain \& Ireland \\
\hline Epipactis sancta & England \\
\hline Erythranthe peregrina & Scotland \\
\hline Euphrasia cambrica & Wales \\
\hline Euphrasia campbelliae & Scotland \\
\hline Euphrasia heslop-harrisonii & Scotland \\
\hline Euphrasia marshallii & Scotland \\
\hline Euphrasia officinalis subsp. anglica & Britain \& Ireland \\
\hline Euphrasia pseudokerneri & Britain \& Ireland \\
\hline Euphrasia rivularis & Britain \\
\hline Euphrasia rotundifolia & Scotland \\
\hline Euphrasia vigursii & England \\
\hline Fumaria capreolata subsp. babingtonii & Britain \& Ireland \\
\hline Fumaria occidentalis & England \\
\hline Fumaria purpurea & Britain \& Ireland \\
\hline Gentianella amarella subsp. anglica & Britain \\
\hline Gentianella amarella subsp. occidentalis & Wales \\
\hline Helianthemum oelandicum subsp. & England \\
\hline
\end{tabular}




\begin{tabular}{|l|l|}
\hline levigatum & \\
\hline Geranium purpureum subsp. forsteri & England and Channel Islands \\
\hline Gymnadenia borealis & Britain \& Ireland but further studies in \\
& NW Europe required \\
\hline Herniaria ciliolata subsp. ciliolata & England and Channel Islands \\
\hline Herniaria ciliolata subsp. subciliata & Channel Islands \\
\hline Hieracium acamptum & England (Extinct) \\
\hline Hieracium aequiserratum & England \\
\hline Hieracium aggregatifolium & Scotland \\
\hline Hieracium aggregatum & Scotland \\
\hline Hieracium amaurostictum & Scotland \\
\hline Hieracium amnicola & England \\
\hline Hieracium ampliatiforme & England \\
\hline Hieracium ampliatum & Britain \& Ireland \\
\hline Hieracium amydrostictum & Scotland \\
\hline Hieracium anfractiforme & Scotland \\
\hline Hieracium anglicum & Britain \& Ireland \\
\hline Hieracium anglorum & Britain \\
\hline Hieracium anguinum & Britain \\
\hline Hieracium angustatiforme & Wales \\
\hline Hieracium angustisquamum & Britain \& Ireland \\
\hline Hieracium apheles & Wales \\
\hline Hieracium aphyllopodioides & Scotland \\
\hline Hieracium apiculatidens & England \\
\hline Hieracium argentatum & Republic of Ireland \\
\hline Hieracium argentiforme & Scotland \\
\hline Hieracium argutifolium & Britain \& Ireland \\
\hline Hieracium aristidens & Scotland \\
\hline Hieracium armadalense & Scotland \\
\hline Hieracium arnsidense & England \\
\hline Hieracium arranense & Scotland \\
\hline Hieracium arvonense & Wales \\
\hline Hieracium ascendentidens & Scotland \\
\hline Hieracium asteridiophyllum & Wales \\
\hline Hieracium atriglandulosum & Scotland \\
\hline Hieracium attenboroughianum & Wales \\
\hline Hieracium attenuatifolium & Scotland \\
\hline Hieracium auratiflorum & Britain \\
\hline Hieracium australius & Scotland \\
\hline Hieracium backhousei & Scotland \\
\hline Hieracium bakerianum & England \\
\hline Hieracium basalticola & Ireland \\
\hline Hieracium basicrinum & Scotland \\
\hline Hieracium beebyanum & Britain \& Ireland \\
\hline Hieracium benhopense & Scotland \\
\hline Hieracium bettyhillense & Scotland \\
\hline
\end{tabular}




\begin{tabular}{|l|l|}
\hline Hieracium boreoanglicum & Britain \\
\hline Hieracium boswellii & Britain \\
\hline Hieracium breacense & Scotland (Extinct) \\
\hline Hieracium breadalbanense & Scotland \\
\hline Hieracium breconense & Wales \\
\hline Hieracium breconicola & Wales \\
\hline Hieracium breve & Scotland \\
\hline Hieracium brigantum & England \\
\hline Hieracium britanniciforme & Britain \\
\hline Hieracium britannicoides & Wales \\
\hline Hieracium britannicum & England \\
\hline Hieracium cacuminum & Wales \\
\hline Hieracium caesiopilosum & Britain \\
\hline Hieracium caesitium & England \\
\hline Hieracium caledonicum & Scotland \\
\hline Hieracium calenduliflorum & Scotland \\
\hline Hieracium callistophyllum & Scotland \\
\hline Hieracium calvum & Scotland \\
\hline Hieracium cambrense & Britain \\
\hline Hieracium cambricogothicum & Wales (Extinct) \\
\hline Hieracium cambricum & Wales \\
\hline Hieracium camptopetalum & Scotland \\
\hline Hieracium candelabrae & England \\
\hline Hieracium cantianum & England \\
\hline Hieracium carenorum & Scotland \\
\hline Hieracium carneddorum & Wales \\
\hline Hieracium centripetale & Scotland \\
\hline Hieracium cerinthiforme & Britain \& Ireland \\
\hline Hieracium charitodon & Wales \\
\hline Hieracium chloranthum & Scotland \\
\hline Hieracium chrysolorum & Britain \\
\hline Hieracium cillense & Wales \\
\hline Hieracium cinderella & Britain \& Ireland \\
\hline Hieracium clivicola & Wales (+England?) \\
\hline Hieracium clovense & Scotland \\
\hline Hieracium completum & Scotland \\
\hline Hieracium cravoniense & Britain \\
\hline Hieracium crebridens & England \\
\hline Hieracium crebridentiforme & England \\
\hline Hieracium cremnanthes & Scotland \\
\hline Hieracium cumbriense & England \\
\hline Hieracium cuneifrons & Britain \\
\hline Hieracium cuspidens & Scotland \\
\hline Hieracium cyathis & Britain \\
\hline Hieracium cyclicum & Britain \& Ireland \\
\hline Hieracium cymbifolium & Britain \\
\hline & \\
\hline
\end{tabular}




\begin{tabular}{|l|l|}
\hline Hieracium daedalolepioides & Britain \\
\hline Hieracium dalense & England \\
\hline Hieracium dasythrix & Scotland \\
\hline Hieracium deargicola & Scotland \\
\hline Hieracium decolor & England \\
\hline Hieracium deganwyense & Britain \& Ireland \\
\hline Hieracium dentulum & Britain \\
\hline Hieracium dewari & Scotland \\
\hline Hieracium dicella & Britain \\
\hline Hieracium difficile & Scotland \\
\hline Hieracium dilectum & Scotland \\
\hline Hieracium dipteroides & Scotland \\
\hline Hieracium discophyllum & Wales \\
\hline Hieracium diversidens & Scotland \\
\hline Hieracium dolorosum & Scotland \\
\hline Hieracium dowardense & Britain \\
\hline Hieracium drummondii & Scotland \\
\hline Hieracium duriceps & Britain $\&$ Ireland \\
\hline Hieracium eboracense & Britain \\
\hline Hieracium ebudicum & Scotland \\
\hline Hieracium einichense & Scotland \\
\hline Hieracium elevatum & Wales \\
\hline Hieracium elongatifolium & Scotland \\
\hline Hieracium eminentiforme & Britain \\
\hline Hieracium erubescens & Britain \& Ireland \\
\hline Hieracium eucallum & Scotland \\
\hline Hieracium eustales & Scotland \\
\hline Hieracium eustomon & Britain \\
\hline Hieracium eximium & Scotland \\
\hline Hieracium farrense & Scotland \\
\hline Hieracium filisquamum & England \\
\hline Hieracium fissuricola & England \\
\hline Hieracium flocculipubens & Britain \& Ireland \\
\hline Hieracium flocculosiforme & Britain \& Ireland \\
\hline Hieracium flocculosum & Britain \& Ireland \\
\hline Hieracium fratrum & Scotland \\
\hline Hieracium fucatifolium & Scotland \\
\hline Hieracium fulvocaesium & Scotland \\
\hline Hieracium glanduliceps & England \\
\hline Hieracium glandulidens & Scotland \\
\hline Hieracium glevense & Britain \\
\hline Hieracium globosiflorum & Scotland \\
\hline Hieracium gothicoides & Scotland \\
\hline Hieracium gracilifolium & Scotland \\
\hline Hieracium grampianum & Scotland \\
\hline Hieracium graniticola & Scotland \\
\hline & \\
\hline
\end{tabular}




\begin{tabular}{|l|l|}
\hline Hieracium gratum & Scotland \\
\hline Hieracium griffithii & Wales (Extinct) \\
\hline Hieracium grovesii & Scotland \\
\hline Hieracium hanburyi & Scotland \\
\hline Hieracium hartii & Republic of Ireland \\
\hline Hieracium hastiforme & Scotland \\
\hline Hieracium hebridense & Britain \& Ireland \\
\hline Hieracium hethlandiae & Scotland (Extinct) \\
\hline Hieracium hibernicum & Ireland \\
\hline Hieracium holophyllum & Britain \\
\hline Hieracium holosericeum & Britain \\
\hline Hieracium hyparcticoides & Scotland \\
\hline Hieracium hypochaeroides & Britain \& Ireland \\
\hline Hieracium hypophalacrum & Scotland \\
\hline Hieracium inaequilaterum & England \\
\hline Hieracium insigne & Scotland \\
\hline Hieracium inspissatum & Wales \\
\hline Hieracium insulare & Scotland \\
\hline Hieracium iricum & Britain \& Ireland \\
\hline Hieracium irregularidens & Scotland \\
\hline Hieracium isabellae & Scotland \\
\hline Hieracium itunense & England \\
\hline Hieracium jaculifolium & England \\
\hline Hieracium jonesianum & England \\
\hline Hieracium kennethii & Scotland \\
\hline Hieracium kentii & England \\
\hline Hieracium kingshousense & Scotland \\
\hline Hieracium kinkellense & Scotland \\
\hline Hieracium kinrossense & Scotland \\
\hline Hieracium kintrawense & Scotland \\
\hline Hieracium klingrahoolense & Scotland \\
\hline Hieracium lacinifolium & England \\
\hline Hieracium laetificum & Scotland \\
\hline Hieracium lagganense & Scotland \\
\hline Hieracium lakelandicum & England \\
\hline Hieracium langwellense & Britain \& Ireland \\
\hline Hieracium larigense & Scotland \\
\hline Hieracium lepiduloides & Britain \& Ireland \\
\hline Hieracium leptodon & Scotland \\
\hline Hieracium leyanum & Britain \\
\hline Hieracium leyi & Britain \\
\hline Hieracium linguans & Wales \\
\hline Hieracium lingulatum & Scotland \\
\hline Hieracium lintonense & Britain \\
\hline Hieracium lintonii & Britain \\
\hline Hieracium maccoshianum & Scotland \\
\hline & \\
\hline
\end{tabular}




\begin{tabular}{|l|l|}
\hline Hieracium macrocarpum & Scotland \\
\hline Hieracium maculoides & England \\
\hline Hieracium magniceps & Scotland (Extinct?) \\
\hline Hieracium mammidens & England \\
\hline Hieracium mariae & Scotland \\
\hline Hieracium maritimum & Britain \& Ireland \\
\hline Hieracium marshallii & Scotland \\
\hline Hieracium megapodium & England \\
\hline Hieracium melanochloricephalum & Scotland \\
\hline Hieracium melanoglochin & Scotland \\
\hline Hieracium memorabile & Scotland \\
\hline Hieracium micracladium & Scotland \\
\hline Hieracium milesii & Scotland \\
\hline Hieracium mirandum & England \\
\hline Hieracium mucronellum & Scotland \\
\hline Hieracium mundum & Scotland \\
\hline Hieracium naviense & England \\
\hline Hieracium neocoracinum & Wales \\
\hline Hieracium neomarginatum & Scotland \\
\hline Hieracium nidense & Wales \\
\hline Hieracium nigrifactum & Scotland \\
\hline Hieracium nitidum & Scotland \\
\hline Hieracium northroense & Scotland \\
\hline Hieracium notabile & Scotland \\
\hline Hieracium obovatifolium & England \\
\hline Hieracium ochthophilum & Scotland \\
\hline Hieracium oenophyllum & Scotland \\
\hline Hieracium optimum & Scotland \\
\hline Hieracium orcadense & Britain \& Ireland \\
\hline Hieracium orimeles & Britain \\
\hline Hieracium orithales & Scotland \\
\hline Hieracium ornatilorum & England \\
\hline Hieracium ovaliforme & Scotland \\
\hline Hieracium oxybeles & Britain \& Ireland \\
\hline Hieracium oxyodus & Scotland (Extinct?) \\
\hline Hieracium pachyphylloides & Britain \\
\hline Hieracium pauculidens & Scotland \\
\hline Hieracium peccense & England \\
\hline Hieracium pensum & Scotland \\
\hline Hieracium pentaploideum & Scotland \\
\hline Hieracium peroblongum & England \\
\hline Hieracium perscitum & Scotland \\
\hline Hieracium perthense & Scotland \\
\hline Hieracium petrocharis & Scotland \\
\hline Hieracium pictorum & Scotland \\
\hline Hieracium piligerum & Britain \\
\hline & \\
\hline
\end{tabular}




\begin{tabular}{|l|l|}
\hline Hieracium placerophylloides & Britain \\
\hline Hieracium pollinarioides & Scotland \\
\hline Hieracium pollinarium & Scotland \\
\hline Hieracium portlandicum & England \\
\hline Hieracium praesigne & Britain \\
\hline Hieracium praethulense & Scotland \\
\hline Hieracium probum & Scotland \\
\hline Hieracium prominentidens & England \\
\hline Hieracium promontoriale & Britain \\
\hline Hieracium protentum & Scotland \\
\hline Hieracium proximum & Britain \& Ireland \\
\hline Hieracium pruinale & Scotland \\
\hline Hieracium pseudacroleucum & Britain \\
\hline Hieracium pseudanglicoides & Scotland \\
\hline Hieracium pseudanglicum & Scotland \\
\hline Hieracium pseudintegratum & England \\
\hline Hieracium pseudocurvatum & Scotland \\
\hline Hieracium pseudoleyi & Wales \\
\hline Hieracium pseudopetiolatum & Scotland \\
\hline Hieracium pseudosarcophyllum & Scotland \\
\hline Hieracium pseudosubcyaneum & England \\
\hline Hieracium pseudozetlandicum & Scotland \\
\hline Hieracium pugsleyi & Scotland \\
\hline Hieracium pulchrius & Britain \\
\hline Hieracium pusillifolium & Wales (Extinct) \\
\hline Hieracium radyrense & Wales \\
\hline Hieracium raveniorum & Scotland \\
\hline Hieracium reayense & Britain \\
\hline Hieracium rectulum & Wales \\
\hline Hieracium repandulare & Wales \\
\hline Hieracium reticulatiforme & Britain \\
\hline Hieracium rhombicum & Britain \\
\hline Hieracium riddelsdellii & Wales \\
\hline Hieracium rivale & Scotland \\
\hline Hieracium robertsii & Wales \\
\hline Hieracium ronasii & Scotland \\
\hline Hieracium ronayense & Scotland \\
\hline Hieracium rubicundiforme & Britain \& Ireland \\
\hline Hieracium rubiginosum & Britain \& Ireland \\
\hline Hieracium sanguineum & Britain \& Ireland \\
\hline Hieracium sannoxense & Scotland \\
\hline Hieracium saxorum & Britain \& Ireland \\
\hline Hieracium scabrisetum & Britain \\
\hline Hieracium scarpicum & Scotland \\
\hline Hieracium scoticum & Scotland \& Ireland \\
\hline Hieracium scottii & Scotland \\
\hline & \\
\hline
\end{tabular}




\begin{tabular}{|l|l|}
\hline Hieracium scullyi & Republic of Ireland \\
\hline Hieracium senescens & Scotland \& Ireland \\
\hline Hieracium shoolbredii & Scotland \\
\hline Hieracium siluriense & Wales \\
\hline Hieracium silvaticoides & Britain \& Ireland \\
\hline Hieracium sinuans & Scotland \\
\hline Hieracium sinuolatum & England \\
\hline Hieracium snowdoniense & Wales \\
\hline Hieracium solum & Scotland \\
\hline Hieracium sommerfeltii & Britain \& Ireland \\
\hline Hieracium sowadeense & Scotland \\
\hline Hieracium sparsifrons & Republic of Ireland \\
\hline Hieracium spenceanum & Scotland \\
\hline Hieracium stenolepiforme & England \\
\hline Hieracium stenopholidium & Britain \\
\hline Hieracium stenophyes & Scotland \\
\hline Hieracium stewartii & Britain \& Ireland \\
\hline Hieracium stictum & England \\
\hline Hieracium strictiforme & Britain \\
\hline Hieracium subamplifolium & Britain \& Ireland \\
\hline Hieracium subbritannicum & Britain \\
\hline Hieracium subcrinellum & Britain \\
\hline Hieracium subcrocatum & Britain \& Ireland \\
\hline Hieracium subcyaneum & England \\
\hline Hieracium subduriceps & Scotland \\
\hline Hieracium subglobosum & Scotland \\
\hline Hieracium subgracilentipes & England \\
\hline Hieracium subhirtum & Scotland \\
\hline Hieracium subintegrifolium & England (Extinct) \\
\hline Hieracium subminutidens & Wales \\
\hline Hieracium submutabile & Britain \\
\hline Hieracium subplanifolium & Britain \\
\hline Hieracium subprasinifolium & England \\
\hline Hieracium subrubicundum & Scotland \& Ireland \\
\hline Hieracium subscoticum & Scotland \\
\hline Hieracium substrigosum & Britain \\
\hline Hieracium subtenue & Scotland \\
\hline Hieracium subtenuifrons & Scotland \\
\hline Hieracium subtruncatum & Scotland \\
\hline Hieracium subumbellatiforme & Scotland \\
\hline Hieracium surrejanum & England \\
\hline Hieracium tavense & Wales \\
\hline Hieracium tenuifrons & Scotland \\
\hline Hieracium thalassinum & Scotland \\
\hline Hieracium tottoense & Scotland \\
\hline Hieracium triangularifolium & Scotland \\
\hline & \\
\hline
\end{tabular}




\begin{tabular}{|l|l|}
\hline Hieracium tricolorans & England \\
\hline Hieracium uiginskyense & Britain \& Ireland \\
\hline Hieracium uistense & Scotland \\
\hline Hieracium uisticola & Scotland \\
\hline $\begin{array}{l}\text { Hieracium umbellatum subsp. } \\
\text { bichlorophyllum }\end{array}$ & Britain \& Ireland \\
\hline Hieracium umbellatum subsp. ogwenii & Wales \\
\hline Hieracium vagense & Britain \\
\hline Hieracium vagicola & England \\
\hline Hieracium varifolium & Scotland \\
\hline Hieracium vennicontium & Britain \\
\hline Hieracium veterascens & Scotland \\
\hline Hieracium vinicaule & Scotland \\
\hline Hieracium vinifolium & Scotland \\
\hline Hieracium vorlichense & Scotland \\
\hline Hieracium westii & Scotland \\
\hline Hieracium zetlandicum & Scotland \\
\hline Limonium binervosum subsp. anglicum & England \\
\hline Limonium binervosum subsp. cantianum & England \\
\hline Limonium binervosum subsp. mutatum & England \\
\hline Limonium binervosum subsp. saxonicum & England \\
\hline $\begin{array}{l}\text { Limonium britannicum subsp. } \\
\text { britannicum }\end{array}$ & England \\
\hline Limonium britannicum subsp. celticum & Britain \\
\hline $\begin{array}{l}\text { Limonium britannicum subsp. } \\
\text { coombense }\end{array}$ & England \\
\hline $\begin{array}{l}\text { Limonium britannicum subsp. } \\
\text { transcanalis }\end{array}$ & Britain \\
\hline Limonium dodartiforme & England \\
\hline Limonium loganicum & England \\
\hline Limonium paradoxum & Wales \\
\hline Limonium parvum & Wales \\
\hline Limonium procerum subsp. cambrense & Wales \\
\hline Limonium procerum subsp. devoniense & England \\
\hline Limonium procerum subsp. procerum & England \\
\hline Limonium recurvum subsp. humile & Britain \\
\hline $\begin{array}{l}\text { Limonium recurvum subsp. } \\
\text { portlandicum }\end{array}$ & Britain \& Ireland \\
\hline $\begin{array}{l}\text { Limonium recurvum subsp. } \\
\text { pseudotranswallianum }\end{array}$ & Republic of Ireland \\
\hline Limonium recurvum subsp. recurvum & England \\
\hline Limonium transwallianum & Wales \\
\hline Linum perenne subsp. anglicum & England \\
\hline Luzula multiflora subsp. hibernica & Ireland \\
\hline Milium vernale subsp. sarniense & Channel Islands \\
\hline Pilosella flagellaris subsp. bicapitata & Scotland \\
\hline
\end{tabular}




\begin{tabular}{|c|c|}
\hline Primula scotica & Scotland \\
\hline Ranunculus acutissimus & England \\
\hline Ranunculus aequilaterus & England \\
\hline Ranunculus angularis & England \\
\hline Ranunculus angustiscutatus & England \\
\hline Ranunculus argillicola & England \\
\hline Ranunculus armingfordensis & England \\
\hline Ranunculus attenuilobus & England \\
\hline Ranunculus brewisiae & England \\
\hline Ranunculus calvitorus & England \\
\hline Ranunculus cantabrigiensis & England \\
\hline Ranunculus combertonensis & England \\
\hline Ranunculus completus & England \\
\hline Ranunculus conspicuidens & England \\
\hline Ranunculus contiguilobus & England \\
\hline Ranunculus convexilobus & England \\
\hline Ranunculus crassilobus & England \\
\hline Ranunculus curvilobus & England \\
\hline Ranunculus dalensis & England \\
\hline Ranunculus densipilus & England \\
\hline Ranunculus dolichodontus & England \\
\hline Ranunculus flammula subsp. minimus & Britain \& Ireland \\
\hline Ranunculus flammula subsp. scoticus & Britain \& Ireland \\
\hline Ranunculus gongylodes & England \\
\hline Ranunculus gracilidens & England \\
\hline Ranunculus gracililobus & England \\
\hline Ranunculus gracilisty/us & England \\
\hline Ranunculus henslowii & England \\
\hline Ranunculus hertfordensis & England \\
\hline Ranunculus horwoodii & England \\
\hline Ranunculus imbricatus & England \\
\hline Ranunculus mammidens & England \\
\hline Ranunculus medioviridis & England \\
\hline $\begin{array}{l}\text { Ranunculus multidens A.C. Leslie, nom. } \\
\text { illeg. }\end{array}$ & England \\
\hline Ranunculus multilobus & England \\
\hline Ranunculus notauricomus & England \\
\hline Ranunculus nubiliviridis & England \\
\hline Ranunculus obesicaulis & England \\
\hline Ranunculus obovatilobatus & England \\
\hline Ranunculus parvidens & England \\
\hline Ranunculus paucipetalus & England \\
\hline Ranunculus petaloideus & England \\
\hline Ranunculus prominentilobus & England \\
\hline Ranunculus pubitorus & England \\
\hline Ranunculus rectilobus & England \\
\hline
\end{tabular}




\begin{tabular}{|l|l|}
\hline Ranunculus rotundilobus & England \\
\hline Ranunculus sepulcralis & England \\
\hline Ranunculus spathulilobus & England \\
\hline Ranunculus stipitatilobus & England \\
\hline Ranunculus stipitatus & England \\
\hline Ranunculus surrejanus & England \\
\hline Ranunculus symmetricidens & England \\
\hline Ranunculus toftensis & England \\
\hline Ranunculus totipetalus & England \\
\hline Ranunculus unipetalus & England \\
\hline Ranunculus vadincisus & England \\
\hline Ranunculus validicaulis & England \\
\hline Ranunculus varipetalus & England \\
\hline Ranunculus viridissimus & England \\
\hline Ranunculus waltersii & England \\
\hline Ranunculus wrayensis & England \\
\hline Rubus acclivitatum & Britain \& Ireland \\
\hline Rubus accrescens & Britain \\
\hline Rubus acutifrons & Britain \\
\hline Rubus adamsii & England \\
\hline Rubus aghadergensis & Britain \& Ireland \\
\hline Rubus ahenifolius & England \\
\hline Rubus albionis & Britain \& Ireland \\
\hline Rubus anglofuscus & Britain \\
\hline Rubus altiarcuatus & Britain, Ireland \& Channel Isles \\
\hline Rubus amplificatus & Britain \& Ireland \\
\hline Rubus angloserpens & England \\
\hline Rubus angusticuspis & Britain \\
\hline Rubus aquarum & Wales \\
\hline Rubus ariconiensis & Britain \& Ireland \\
\hline Rubus aristisepalus & Britain \\
\hline Rubus armipotens & Britain \\
\hline Rubus avaloniensis & England \\
\hline Rubus averyanus & England \\
\hline Rubus babingtonianus & England \\
\hline Rubus babingtonii & Britain \\
\hline Rubus bagnallianus & England \\
\hline Rubus bakerianus & England \\
\hline Rubus bartonii & Britain \& Ireland \\
\hline Rubus biloensis & Britain \\
\hline Rubus bloxamianus & England \\
\hline Rubus boudiccae & Britain \& Ireland \\
\hline Rubus breconensis & Wales \\
\hline Rubus brevistaminosus & England \\
\hline Rubus briggsianus & Britain, Ireland, Isle of Man, Channel \\
& Isles \\
\hline & \\
\hline
\end{tabular}




\begin{tabular}{|l|l|}
\hline Rubus briggsii & England \\
\hline Rubus britannicus & England \\
\hline Rubus bucknallii & England \\
\hline Rubus calvatus & Britain \& Ireland \\
\hline Rubus cambrensis & Britain \\
\hline Rubus canterburiensis & England \\
\hline Rubus carnkiefensis & England \\
\hline Rubus castrensis & England \\
\hline Rubus celticus & Wales (Ireland?) \\
\hline Rubus cerdicii & England \\
\hline Rubus chrysoxylon & Britain \\
\hline Rubus cinerosiformis & Britain \& Ireland \\
\hline Rubus cinerosus & England \& Ireland \\
\hline Rubus clausentinus & England \\
\hline Rubus confertiflorus & England \\
\hline Rubus conspersus & England \\
\hline Rubus cordatifolius & Channel Islands \\
\hline Rubus cornubiensis & England \\
\hline Rubus cotteswoldensis & England \\
\hline Rubus crespignyanus & England \\
\hline Rubus criniger & England \& Isle of Man \\
\hline Rubus crudelis & England \\
\hline Rubus cumbrensis & Britain \\
\hline Rubus Curvidens & Britain \\
\hline Rubus curvispinosus & England \\
\hline Rubus daltrii & England \\
\hline Rubus dasycoccus & Britain \\
\hline Rubus daveyi & England and Channel Islands \\
\hline Rubus decussatus & England \\
\hline Rubus devoniensis & England \\
\hline Rubus distractiformis & Britain \& Ireland \\
\hline Rubus diversiarmatus & England \\
\hline Rubus dobuniensis & England (Extinct?) \\
\hline Rubus dunensis & Britain \& Ireland \\
\hline Rubus durescens & England \\
\hline Rubus durotrigum & England \\
\hline Rubus eboracensis & Britain \\
\hline Rubus ebudensis & Scotland \\
\hline Rubus echinatoides & Britain \& Ireland \\
\hline Rubus effrenatus & Britain (Ireland?) \\
\hline Rubus errabundus & Britain \& Ireland \\
\hline Rubus erythrops & England \\
\hline Rubus euanthinus & Britain \\
\hline Rubus furnarius & England \\
\hline Rubus furvicolor & Scotland \\
\hline Rubus fuscicaulis & Britain \& Ireland \\
\hline & \\
\hline
\end{tabular}




\begin{tabular}{|l|l|}
\hline Rubus fuscicortex & Britain \\
\hline Rubus gallofuscus & Britain \\
\hline Rubus gariannensis & England \\
\hline Rubus glanduliger & England \\
\hline Rubus griffithianus & Britain \& Ireland \\
\hline Rubus halsteadensis & Britain \\
\hline Rubus hantonensis & England \\
\hline Rubus hebridensis & Britain \& Ireland \\
\hline Rubus herefordensis & England \\
\hline Rubus hesperius & Ireland \& Isle of Man \\
\hline Rubus heterobelus & England \\
\hline Rubus hibernicus & Britain \& Ireland \\
\hline Rubus hindii & Britain \& Ireland \\
\hline Rubus hirsutissimus & Britain \\
\hline Rubus hylocharis & Britain \& Ireland \\
\hline Rubus hyposericeus & England \\
\hline Rubus iceniensis & England \\
\hline Rubus incurvatiformis & Britain \\
\hline Rubus infestior & England \\
\hline Rubus infestisepalus & England \\
\hline Rubus informifolius & England \\
\hline Rubus intensior & Britain \\
\hline Rubus iodnephes & England \\
\hline Rubus iricus & Britain \& Ireland, Channel Isles \\
\hline Rubus iscanus & Britain \\
\hline Rubus lacustris & England \\
\hline Rubus lamburnensis & Britain \& Ireland, Channel Isles \\
\hline Rubus largificus & Britain \& Ireland \\
\hline Rubus lasiodermis & England \\
\hline Rubus latifolius & Britain \& Ireland \\
\hline Rubus laxatifrons & England \\
\hline Rubus lentiginosus & Britain \& Ireland \\
\hline Rubus lettii & Britain \& Ireland \\
\hline Rubus leucandriformis & England \\
\hline Rubus lintonii & England \\
\hline Rubus londinensis & England \\
\hline Rubus longifrons & England (Extinct?) \\
\hline Rubus longus & Britain \& Ireland \\
\hline Rubus ludensis & Britain \\
\hline Rubus malvernicus & Britain \& Ireland \\
\hline Rubus marshallii & England \\
\hline Rubus melanocladus & Britain \\
\hline Rubus mercicus & England \\
\hline Rubus merlinii & Wales \\
\hline Rubus metallorum & England (if native; introduced in \\
\hline Rubus milesianus & \\
\hline
\end{tabular}




\begin{tabular}{|l|l|}
\hline & Ireland) \\
\hline Rubus mollissimus & England \\
\hline Rubus monensis & Wales \\
\hline Rubus morganwgensis & Britain \\
\hline Rubus moylei & Britain \& Ireland \\
\hline Rubus mucronatiformis & England (Ireland?) \\
\hline Rubus mucronatoides & Britain \\
\hline Rubus newbouldianus & England \\
\hline Rubus newbouldii & Britain \\
\hline Rubus newbridgensis & England \\
\hline Rubus newtonii & Britain \\
\hline Rubus norvicensis & Britain \& Ireland, Channel Isles \\
\hline Rubus obesifolius & England \\
\hline Rubus obscuriflorus & England \\
\hline Rubus orbus & Britain \\
\hline Rubus ordovicum & Britain \& Ireland \\
\hline Rubus painteri & England \& Ireland \\
\hline Rubus pallidisetus & Britain \& Ireland \\
\hline Rubus pampinosus & Britain \\
\hline Rubus pascuorum & Britain \& Ireland \\
\hline Rubus percrispus & Britain \& Ireland, Channel Isles \\
& (Extinct?) \\
\hline Rubus perdigitatus & Britain \\
\hline Rubus permundus & England \\
\hline Rubus pervalidus & England \\
\hline Rubus pictorum & Britain \\
\hline Rubus pliocenicus & England \\
\hline Rubus poliodes & England \\
\hline Rubus polyoplus & Britain \& Ireland \\
\hline Rubus porphyrocaulis & England \\
\hline Rubus powellii & England \\
\hline Rubus pseudoplinthostylus & England \\
\hline Rubus pullifolius & England \\
\hline Rubus purchasianus & Britain \\
\hline Rubus putneiensis & England \\
\hline Rubus pydarensis & England and Channel Islands \\
\hline Rubus regillus & England \\
\hline Rubus riddelsdellii & Britain \& Ireland \\
\hline Rubus robiae & Britain \& Ireland \\
\hline Rubus rossensis & Britain \& Ireland \\
\hline Rubus rotundifolius & England (Extinct?) \\
\hline Rubus rubriflorus & England \\
\hline Rubus rubristylus & Britain \& Ireland, Isle of Man \\
\hline Rubus sagittarius & England \\
\hline Rubus salteri & England \\
\hline Rubus scoticus & Britain \& Ireland \\
\hline & \\
\hline
\end{tabular}




\begin{tabular}{|l|l|}
\hline Rubus sectiramus & England \\
\hline Rubus segontii & Britain \& Ireland \\
\hline Rubus semiglaber & Wales \\
\hline Rubus silurum & Britain (Ireland?) \\
\hline Rubus sneydii & England \\
\hline Rubus spadix & England \\
\hline Rubus stanneus & England \\
\hline Rubus subintegribasis & England \\
\hline Rubus surrejanus & Britain (introduced to Ireland?) \\
\hline Rubus tardus & England \\
\hline Rubus tavensis & Wales \\
\hline Rubus tenuiarmatus & Britain \\
\hline Rubus trelleckensis & Wales \\
\hline Rubus tresidderi & England \\
\hline Rubus triangularis & England \\
\hline Rubus trinovantium & England \\
\hline Rubus troiensis & Britain \\
\hline Rubus turritus & England \\
\hline Rubus vagensis & Britain \\
\hline Rubus varvicensis & England \\
\hline Rubus villicauliformis & Britain, Ireland and Channel Islands \\
\hline Rubus villosior & England \\
\hline Rubus vindomensis & England \\
\hline Rubus waddellii & Ireland \& Isle of Man \\
\hline Rubus warrenii & Britain \& Ireland \\
\hline Rubus watsonii & England \\
\hline Rubus wedgwoodiae & England \\
\hline Rubus wirralensis & Britain, Ireland \& Isle of Man \\
\hline Rubus wolley-dodii & England \\
\hline Rumex acetosa subsp. hibernicus & Britain \& Ireland \\
\hline Saxifraga rosacea subsp. hartii & Republic of Ireland \\
\hline Scleranthus perennis subsp. prostratus & England \\
\hline Senecio cambrensis & Britain \\
\hline Senecio eboracensis & England (Extinct) \\
\hline Sisyrinchium bermudiana & Ireland (if different to North American \\
taxon; naturalised in Britain)
\end{tabular}




\begin{tabular}{|l|l|}
\hline Sorbus eminens & Britain \\
\hline Sorbus eminentiformis & Britain \\
\hline Sorbus eminentoides & England \\
\hline Sorbus evansii & England \\
\hline Sorbus greenii & England \\
\hline Sorbus herefordensis & England \\
\hline Sorbus hibernica & Ireland \\
\hline Sorbus lancastriensis & England \\
\hline Sorbus leighensis & England \\
\hline Sorbus leptophylla & Wales \\
\hline Sorbus leyana & Wales \\
\hline Sorbus margaretae & England \\
\hline Sorbus minima & Wales \\
\hline Sorbus parviloba & England \\
\hline Sorbus porrigentiformis (including S. & Britain \\
\hline humphreyana) & \\
\hline Sorbus pseudofennica & Scotland \\
\hline Sorbus pseudomeinichii & Scotland \\
\hline Sorbus richii & England \\
\hline Sorbus rupicoloides & England \\
\hline Sorbus saxicola & England \\
\hline Sorbus scannelliana & Republic of Ireland \\
\hline Sorbus spectans & England \\
\hline Sorbus stenophylla & Wales \\
\hline Sorbus stirtoniana & Wales \\
\hline Sorbus subcuneata & England \\
\hline Sorbus vexans & England \\
\hline Sorbus whiteana & England \\
\hline Sorbus wilmottiana & England \\
\hline Spartina anglica & Britain but widely planted elsewhere \\
\hline Taraxacum acutum & England \\
\hline Taraxacum aesculosum & Britain \& Ireland \\
\hline Taraxacum amarellum & Republic of Ireland \\
\hline Taraxacum amicorum & England \\
\hline Taraxacum atrocollinum & Britain \& Ireland \\
\hline Taraxacum berthae & Britain \\
\hline Taraxacum breconense & Wales \\
\hline Taraxacum caledonicum & Scotland \\
\hline Taraxacum calophyllum & Scotland \\
\hline Taraxacum cambricum & Britain \& Ireland \\
\hline Taraxacum celticum & Britain \& Ireland \\
\hline Taraxacum cherwellense & England \\
\hline Taraxacum chrysoglossum & Scotland \\
\hline Taraxacum claudiae & Scotland \\
\hline Taraxacum clovense & Scotland \\
\hline Taraxacum cophocentrum & Britain \& Ireland \\
\hline & \\
\hline
\end{tabular}




\begin{tabular}{|l|l|}
\hline Taraxacum cornubiense & England \\
\hline Taraxacum degelii & Britain \& Ireland \\
\hline Taraxacum elegantifrons & Republic of Ireland \\
\hline Taraxacum fulvicarpum & Britain \& Ireland \\
\hline Taraxacum gaelorum & Republic of Ireland \\
\hline Taraxacum geirhildae & Scotland \\
\hline Taraxacum haworthianum & Britain \& Ireland \\
\hline Taraxacum hesperium & Britain \& Ireland \\
\hline Taraxacum hexhamense & Britain \& Ireland \\
\hline Taraxacum hibernicola & Republic of Ireland \\
\hline Taraxacum hirsutissimum & Scotland \\
\hline Taraxacum inane & Britain \& Ireland \\
\hline Taraxacum inclinorum & Republic of Ireland \\
\hline Taraxacum inopinatum & Britain \& Ireland \\
\hline Taraxacum lancastriense & Britain \\
\hline Taraxacum luteum & Britain \& Ireland \\
\hline Taraxacum margettsii & England \\
\hline Taraxacum nigridentatum & Britain \\
\hline Taraxacum oellgaardii & Britain \\
\hline Taraxacum olgae & Britain \\
\hline Taraxacum orcadense & Scotland \\
\hline Taraxacum palmeri & Scotland \\
\hline Taraxacum palustrisquameum & Britain \& Ireland \\
\hline Taraxacum pankhurstianum & Scotland \\
\hline Taraxacum porteri & Britain \\
\hline Taraxacum pseudonordstedtii & England \\
\hline Taraxacum richardsianum & Britain \\
\hline Taraxacum ronae & Britain \& Ireland \\
\hline Taraxacum rufofructum & Scotland \\
\hline Taraxacum sahlinianum & Britain \& Ireland \\
\hline Taraxacum scoticum & Britain \\
\hline Taraxacum serpenticola & Scotland \\
\hline Taraxacum stenacrum & Britain \& Ireland \\
\hline Taraxacum subbracteatum & Britain \& Ireland \\
\hline Taraxacum subnaevosum & Britain \\
\hline Taraxacum tanylepis & Scotland \\
\hline Taraxacum webbii & Republic of Ireland \\
\hline Tephroseris integrifolia subsp. maritima & Wales \\
\hline Ulmus acuminatissima & England \\
\hline Ulmus acutissima & England \\
\hline Ulmus alta & England \\
\hline Ulmus anglosaxonica & England \\
\hline Ulmus angustiformis & Britain \\
\hline Ulmus asymmetrica & England \\
\hline Ulmus atrovirens & England \\
\hline Ulmus cantabrigiensis & England \\
\hline & \\
\hline
\end{tabular}




\begin{tabular}{|l|l|}
\hline Ulmus chaterorum & Wales \\
\hline Ulmus coriaceifolia & England \\
\hline Ulmus coritana & England \\
\hline Ulmus cornubiensis & England \\
\hline Ulmus crassa & England \\
\hline Ulmus crenata & England \\
\hline Ulmus cuneiformis & Britain \\
\hline Ulmus curvifolia & England \\
\hline Ulmus daveyi & Britain \\
\hline Ulmus diversifolia & England \\
\hline Ulmus elegantissima & Britain \\
\hline Ulmus exoniensis & England \\
\hline Ulmus gyrophylla & Britain \\
\hline Ulmus hollandica & Britain \\
\hline Ulmus incisa & England \\
\hline Ulmus longicaudata & Britain \\
\hline Ulmus longidens & Britain \\
\hline Ulmus longidentata & England \\
\hline Ulmus madingleyensis & England \\
\hline Ulmus microdon & England \\
\hline Ulmus minor & England \\
\hline Ulmus mossii & Britain \\
\hline Ulmus multidentata & England \\
\hline Ulmus obesidens & England \\
\hline Ulmus oblanceolata & England \\
\hline Ulmus occidentalis & Britain \\
\hline Ulmus peninsularis & England \\
\hline Ulmus platyphylla & England \\
\hline Ulmus plotii & Britain \\
\hline Ulmus prionophylla & England \\
\hline Ulmus prominentidens & England \\
\hline Ulmus pseudelegantissima & England \\
\hline Ulmus pseudobovata & England \\
\hline Ulmus pseudocoritana & England \\
\hline Ulmus rasilis & England \\
\hline Ulmus rhombifolia & England \\
\hline Ulmus sativa & England \\
\hline Ulmus serrata & England \\
\hline Ulmus serratifrons & England \\
\hline Ulmus serratula & England \\
\hline Ulmus sowerbyi & England \\
\hline Ulmus sy/vatica & England \\
\hline Ulmus vegeta & Britain \\
\hline Ulmus wheatleyi & Britain \\
\hline & \\
\hline
\end{tabular}

